\title{
Direktwahl des EU-Kommissionspräsidenten: Mehr Schaden als Nutzen
}

VB

verfassungsblog.de/direktwahl-des-eukommissionsprsidenten-mehr-schaden-als-nutzen/

Christian Joerges , Florian Rödl Di 26 Jun 2012

Di 26 Jun

2012

Von CHRISTIAN JOERGES und FLORIAN RÖDL

Mattias Kumm hat in diesem Blog kürzlich Vorschläge vorgelegt, wie die demokratische Legitimation der EU im Angesicht der Eurokrise gestärkt werden könnte, ohne dass man dazu die europäischen Verträge ändern muss. Die Vorschläge gehen auf einen Policy-Report zum Thema "democratic goverance of the Euro" (zu deutsch vielleicht: „demokratisches Regieren des Euro"?) zurück, den Kumm und zwei weitere renommierte Kollegen am Europäischen Hochschulinstitut in Florenz, Bruno de Witte und Miguel Poiares Maduro, verfasst haben.[1] Wir halten diese Vorschläge nicht für überzeugend.

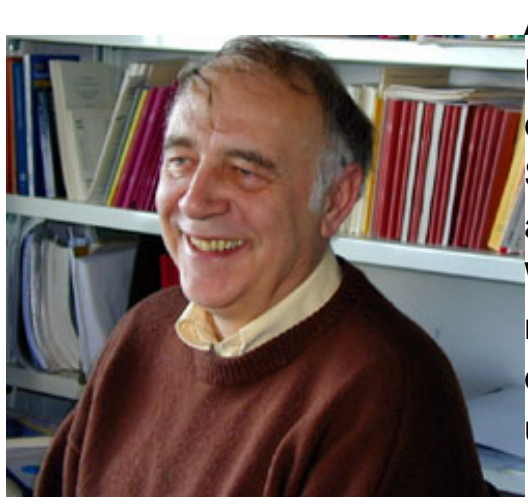

Aus dem Vorspann des Reports geht hervor, dass Anlass für seine Erstellung die Überzeugung war, dass Europa gerade auch mit Bezug auf den Euro demokratische Defizite aufweist. Betont wird dabei, dass nach dem erreichten Stand der Integration die Politik eines Mitgliedstaates Auswirkungen auf alle anderen Mitgliedstaaten habe. Das soll gerade für die mitgliedstaatliche Wirtschafts- und Finanzpolitik gelten: Den Kern der Krise mache ein mitgliedstaatliches Demokratieversagen aus. Nicht benannt wird demgegenüber die demokratische Involution, der die Union und ihre Mitgliedstaaten gegenwärtig unterzogen werden. Das ist erstaunlich und befremdlich.

Gleiches gilt für die Kernvorschläge, die der vorgestellte Policy-Report enthält. Der erste Vorschlag ist deckungsgleich mit einer von den Führungseliten der europäischen Sozialdemokratie bereits eingeschlagenen Strategie, zu der auch die personellen Weichen bereits gestellt sind: Zur Europawahl sollen die europäischen Parteiverbünde mit einem europäischen Spitzenkandidaten antreten (für die SPE: Martin Schulz). Der Gewinner der relativen Mehrheit für seine Partei soll das Amt des Kommissionspräsidenten beanspruchen, auch wenn das Vorschlagsrecht an sich dem Europäischen Rat zusteht. Der zweite Vorschlag, insgesamt etwas vager gehalten, empfiehlt eine stärkere Regulierung der Finanzmärkte, darunter die Einführung einer Finanztransaktionssteuer, deren Erträge überdies der EU als Eigenmittel zufließen sollen. Auch hiermit ist ein Punkt der seit Längerem verfolgten europapolitischen Agenda der Sozialdemokratie aufgegriffen.

Wir halten beide Vorschläge für wenig überzeugend, weil sie der Problemlage der Union nicht gerecht werden. Diese Problemlage ist in der Tat von einem Demokratiedefizit innerhalb der Union geprägt. Aber die externen Effekte, die der mitgliedstaatlichen Politik zugerechnet werden, sind im Zusammenhang mit der Währungsunion allenfalls ein Oberflächenphänomen. Dies gilt schon deswegen, weil diese Effekte wesentlich dem Umstand geschuldet sind, dass die Währungsunion die makroökonomischen Handlungsmöglichkeiten der Mitgliedstaaten so drastisch eingeschränkt und ihnen zusätzlich die überaus schwierige Aufgabe wirtschaftlicher Konvergenz aufgebürdet hat.

Der Fokus auf das bloße Oberflächenphänomen führt bei der Suche nach der Kur der Problematik völlig in die Irre. Wir konzentrieren uns im Folgenden auf den Vorschlag, die demokratische Legitimation des „Regierens des Euro“ dadurch zu stärken, dass die EP-

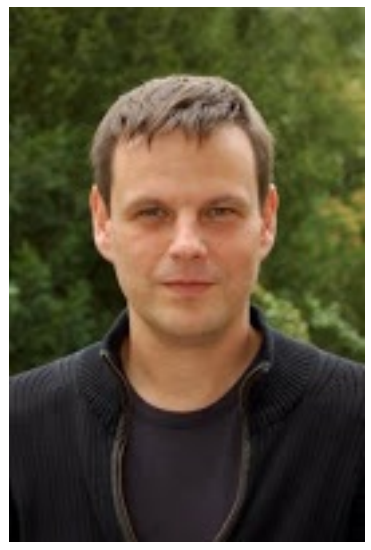
Wahl künftig zugleich eine Wahl des Kommissionspräsidenten darstellen soll, und lassen Finanztransaktionssteuer etc. außen vor. 
In der gegenwärtigen Verfassung der Wirtschafts- und Währungsunion nimmt aus systemischen Gründen die Notwendigkeit zu, dass mitgliedstaatliche Politik durch die europäische Ebene korrigiert wird. Denn der Euro verlangt einerseits die wirtschaftliche Konvergenz der Mitgliedstaaten, andererseits treibt er die mitgliedstaatlichen Ökonomien mit einheitlichem Außenwechselkurs, einheitlichem Zinssatz und - dank „Six Pack“ und „Fiskalpakt“ alsbald auch effektiv - vereinheitlichter Budgetpolitik auseinander. Die Mitgliedstaaten sind aber augenscheinlich nicht in der Lage oder auch nicht willens, die aus dieser Konstellation resultierenden makroökonomischen Imperative möglichst ohne Abstriche als Resultate demokratischer Politik auszuwerfen. Weil die Diskrepanzen nicht nur den Mitgliedstaat allein, sondern Eurozone und EU insgesamt betreffen, muss die Unionsebene den makroökonomischen Imperativen zur Durchsetzung verhelfen. Weil aber die Unionsebene angesichts der Schlüsselstellung des Rates allzu stark durch die Repräsentation der zu korrigierenden Mitgliedstaaten geprägt ist, muss das institutionelle Gleichgewicht innerhalb des politischen Systems der Union zugunsten der Kommission verschoben werden.

Es ist wichtig zu sehen: Diese Dynamik der Währungsunion bedeutet insgesamt keine Vertiefung der Integration im bisherigen Modus der Harmonisierung, also der Formung der unterschiedlichen mitgliedstaatlichen

Rechtsordnungen durch europäische Rechtssetzung bis hin zur Vereinheitlichung. Vielmehr geht es beim „Regieren des Euro" um eine situative kommissarische Beaufsichtigung mitgliedstaatlicher Demokratien gerade auch in den Bereichen, die kompetenziell weiterhin auf mitgliedstaatlicher Ebene angesiedelt sind und auch bleiben sollen.

All dies hält der Policy-Report augenscheinlich nicht für problematisch. Das wird den Beobachter der verfassungsrechtlichen Kontroversen ebenso erstaunen wie den des politischen Tagesgeschehens in den Ländern Griechenland, Portugal, Spanien oder Italien. Freilich: Wenn man den Unterschied von supranationaler Integration durch Recht und situativer kommissarischer Aufsicht tatsächlich nicht in den Blick nimmt oder für unproblematisch hält, dann kann die Frage nach möglicher Demokratisierung gar nicht anders beantwortet werden als mit der Wahl des kommissarischen Aufsehers, also mit der Direktwahl des Kommissionspräsidenten, dem nach heutigen Stand einzigen originär auf Unionsebene bestimmten Mitglied der Kommission. Weil jedoch die echte Direktwahl eine Vertragsänderung erforderte, bleibt das Votum für eine „als ob“-Direktwahl durch Spitzenkandidaten mit entsprechenden Ansprüchen.

Der Gedanke ist verwegen: Die Unionsbürger sollen repräsentiert durch das EP mit dem Kommissionpräsidenten eine Führungsfigur selbst wählen, die immer mehr den Mitgliedstaaten und deren Staatsbürgern autoritativ vorgeben soll, welche Ergebnisse die mitgliedstaatliche demokratische Politik haben kann und darf. So bleibt es zwar bei der kommissarischen Beaufsichtigung mitgliedstaatlicher Demokratien, die sich nun aber dadurch legitimieren soll, dass die Leitfigur der Aufsichtsbehörde demokratisch gewählt wird. Sollen wir wirklich glauben, dass die Bürger in den betroffenen Mitgliedstaaten, vor allem diejenigen, die von massiver Lohnsenkung und Schleifung sozialstaatlicher Sicherung existentiell betroffen sind - dies gehört bekanntlich zu den wesentlichen makroökonomischen Imperativen des „Regierens des Euro“ - davon wirklich zu beeindrucken sind? Wir befürchten demgegenüber, dass in dieser Konstellation ein Verweis auf eine neue „demokratische Legitimation“ des Kommissionpräsidenten von den betroffenen Bürgern, die sich in der heutigen Lage der Griechen, Portugiesen oder Spanier befinden, schlicht als Hohn empfunden würde.

Natürlich gibt es noch weitere Einwände gegen die (informelle) Direktwahl des Kommissionspräsidenten, die der Policy-Report nicht anspricht.

- Eine Wahl ohne Wahlrechtsgleichheit wird keine Akzeptanz finden, und zwar aus guten theoretischen Gründen, wenn denn der bislang gültige Grundsatz der rechtsstaatlichen Demokratietheorie, dass nämlich der Zwang des Rechts seine Legitimation aus der gleichberechtigen Teilhabe an der Genese des Rechts bezieht, nicht revidiert werden soll (oder wenn er denn revidiert werden soll, wie es allenthalben propagiert wird, wäre dies wenigstens einmal in theoria zu leisten).

- Die alteuropäische Demokratietradition sorgt sich vor allem um die rechtliche Bindung der Exekutive durch parlamentarisches Gesetz und steht darum der direkten Wahl exekutiver Spitzen generell skeptisch 
gegenüber. Die Bedenken gegenüber einer identitären Repräsentation durch eine Wahl der Spitze der Exekutive sind umso berechtigter, wenn die demokratischen Gegengewichte - Parlament, Parteien, Verbände, Öffentlichkeit - so schwach entwickelt sind wie in der Union.

- Unter den gegebenen Umständen würden zwar Konservative und Sozialdemokraten um das Amt des Kommissionpräsidenten konkurrieren. Aber die Wählerschaft würde sich nicht entlang der üblichen RechtsLinks-Achse organisieren, sondern entlang der Wohlstandsachse Nord-Süd. Schließlich ist - salopp gesprochen - nach Meinung auch vieler Sozialdemokraten Austeritätspolitik nicht schlecht, wenn sie die anderen trifft und die Haftungsrisiken des eigenen Mitgliedstaates zu senken verspricht. Statt demokratischer Legitimation würde die Direktwahl dann eine tiefe soziale Spaltung der Union ins Werk setzen, und der „demokratisch legitimierte“ Kommissionspräsident würde als nichts anderes denn als Repräsentant dieser Spaltung angesehen werden.

Wir sehen von einer Verlängerung dieser Reihe ab.

[1] Policy-Report "The Euro Crisis and the Democratic Governance of the Euro: Legal and Political Issues of a Fiscal Crisis”, vorgelegt für das High-Level Policy Seminar "The Democratic Governance of the Euro" am 10.5.2012 (alsbald verfügbar unter http://globalgovernanceprogramme.eui.eu/news-events/high-level-policy-seminars/thedemocratic-governance-of-the-euro/). Zu diesem Seminar wurden in einem Compiled Memorandum weitere Stellungnahmen veröffentlicht, darunter auch ein Beitrag von Ch. Joerges („The Economic Constitution in Crisis“).

Prof. Dr. Christian Joerges ist Direktor am Zentrum für Europäische Rechtspolitik und Professor für Deutsches und Europäisches Privat- und Wirtschaftsrecht an der Universität Bremen. Dr. Florian Rödl ist Forschungsgruppenleiter am Excellenzcluster "Die Herausbildung normativer Ordnungen" der Goethe-Universität Frankfurt/Main.

\section{Von CHRISTIAN JOERGES und FLORIAN RÖDL}

Mattias Kumm hat in diesem Blog kürzlich Vorschläge vorgelegt, wie die demokratische Legitimation der EU im Angesicht der Eurokrise gestärkt werden könnte, ohne dass man dazu die europäischen Verträge ändern muss. Die Vorschläge gehen auf einen Policy-Report zum Thema „democratic goverance of the Euro“ (zu deutsch vielleicht: „demokratisches Regieren des Euro“?) zurück, den Kumm und zwei weitere renommierte Kollegen am Europäischen Hochschulinstitut in Florenz, Bruno de Witte und Miguel Poiares Maduro, verfasst haben.[1] Wir halten diese Vorschläge nicht für überzeugend.

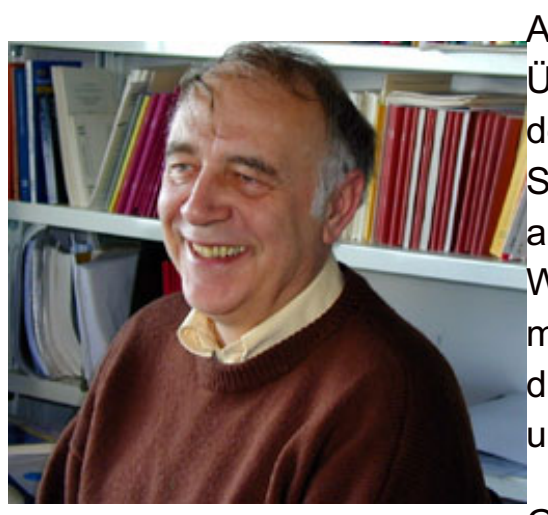

Aus dem Vorspann des Reports geht hervor, dass Anlass für seine Erstellung die Überzeugung war, dass Europa gerade auch mit Bezug auf den Euro demokratische Defizite aufweist. Betont wird dabei, dass nach dem erreichten Stand der Integration die Politik eines Mitgliedstaates Auswirkungen auf alle anderen Mitgliedstaaten habe. Das soll gerade für die mitgliedstaatliche Wirtschafts- und Finanzpolitik gelten: Den Kern der Krise mache ein mitgliedstaatliches Demokratieversagen aus. Nicht benannt wird demgegenüber die demokratische Involution, der die Union und ihre Mitgliedstaaten gegenwärtig unterzogen werden. Das ist erstaunlich und befremdlich.

Gleiches gilt für die Kernvorschläge, die der vorgestellte Policy-Report enthält. Der erste Vorschlag ist deckungsgleich mit einer von den Führungseliten der europäischen Sozialdemokratie bereits eingeschlagenen Strategie, zu der auch die personellen Weichen bereits gestellt sind: Zur Europawahl sollen die europäischen Parteiverbünde mit einem europäischen Spitzenkandidaten antreten (für die SPE: Martin Schulz). Der Gewinner der relativen Mehrheit für seine Partei soll das Amt des Kommissionspräsidenten beanspruchen, auch wenn das Vorschlagsrecht an sich dem Europäischen Rat zusteht. Der zweite Vorschlag, insgesamt etwas vager gehalten, empfiehlt eine stärkere Regulierung der Finanzmärkte, darunter die Einführung einer Finanztransaktionssteuer, deren Erträge überdies der EU als Eigenmittel zufließen sollen. Auch hiermit ist ein Punkt 
der seit Längerem verfolgten europapolitischen Agenda der Sozialdemokratie aufgegriffen.

Wir halten beide Vorschläge für wenig überzeugend, weil sie der Problemlage der Union nicht gerecht werden. Diese Problemlage ist in der Tat von einem Demokratiedefizit innerhalb der Union geprägt. Aber die externen Effekte, die der mitgliedstaatlichen Politik zugerechnet werden, sind im Zusammenhang mit der Währungsunion allenfalls ein Oberflächenphänomen. Dies gilt schon deswegen, weil diese Effekte wesentlich dem Umstand geschuldet sind, dass die Währungsunion die makroökonomischen Handlungsmöglichkeiten der Mitgliedstaaten so drastisch eingeschränkt und ihnen zusätzlich die überaus schwierige Aufgabe wirtschaftlicher Konvergenz aufgebürdet hat.

Der Fokus auf das bloße Oberflächenphänomen führt bei der Suche nach der Kur der Problematik völlig in die Irre. Wir konzentrieren uns im Folgenden auf den Vorschlag, die demokratische Legitimation des „Regierens des Euro“ dadurch zu stärken, dass die EPWahl künftig zugleich eine Wahl des Kommissionspräsidenten darstellen soll, und lassen Finanztransaktionssteuer etc. außen vor.

In der gegenwärtigen Verfassung der Wirtschafts- und Währungsunion nimmt aus systemischen Gründen die Notwendigkeit zu, dass mitgliedstaatliche Politik durch die europäische Ebene korrigiert wird. Denn der Euro verlangt einerseits die wirtschaftliche Konvergenz der Mitgliedstaaten, andererseits treibt er die mitgliedstaatlichen Ökonomien mit einheitlichem Außenwechselkurs, einheitlichem Zinssatz und - dank "Six Pack“ und „Fiskalpakt“ alsbald auch effektiv - vereinheitlichter Budgetpolitik auseinander. Die Mitgliedstaaten sind aber augenscheinlich nicht in der Lage oder auch nicht willens, die aus dieser Konstellation resultierenden makroökonomischen Imperative möglichst ohne Abstriche als Resultate demokratischer Politik auszuwerfen. Weil die Diskrepanzen nicht nur den Mitgliedstaat allein, sondern Eurozone und EU insgesamt betreffen, muss die Unionsebene den makroökonomischen Imperativen zur Durchsetzung verhelfen. Weil aber die Unionsebene angesichts der Schlüsselstellung des Rates allzu stark durch die Repräsentation der zu korrigierenden Mitgliedstaaten geprägt ist, muss das institutionelle Gleichgewicht innerhalb des politischen Systems der Union zugunsten der Kommission verschoben werden.

Es ist wichtig zu sehen: Diese Dynamik der Währungsunion bedeutet insgesamt keine Vertiefung der Integration im bisherigen Modus der Harmonisierung, also der Formung der unterschiedlichen mitgliedstaatlichen

Rechtsordnungen durch europäische Rechtssetzung bis hin zur Vereinheitlichung. Vielmehr geht es beim „Regieren des Euro" um eine situative kommissarische Beaufsichtigung mitgliedstaatlicher Demokratien gerade auch in den Bereichen, die kompetenziell weiterhin auf mitgliedstaatlicher Ebene angesiedelt sind und auch bleiben sollen.

All dies hält der Policy-Report augenscheinlich nicht für problematisch. Das wird den Beobachter der verfassungsrechtlichen Kontroversen ebenso erstaunen wie den des politischen Tagesgeschehens in den Ländern Griechenland, Portugal, Spanien oder Italien. Freilich: Wenn man den Unterschied von supranationaler Integration durch Recht und situativer kommissarischer Aufsicht tatsächlich nicht in den Blick nimmt oder für unproblematisch hält, dann kann die Frage nach möglicher Demokratisierung gar nicht anders beantwortet werden als mit der Wahl des kommissarischen Aufsehers, also mit der Direktwahl des Kommissionspräsidenten, dem nach heutigen Stand einzigen originär auf Unionsebene bestimmten Mitglied der Kommission. Weil jedoch die echte Direktwahl eine Vertragsänderung erforderte, bleibt das Votum für eine „als ob“-Direktwahl durch Spitzenkandidaten mit entsprechenden Ansprüchen.

Der Gedanke ist verwegen: Die Unionsbürger sollen repräsentiert durch das EP mit dem Kommissionpräsidenten eine Führungsfigur selbst wählen, die immer mehr den Mitgliedstaaten und deren Staatsbürgern autoritativ vorgeben soll, welche Ergebnisse die mitgliedstaatliche demokratische Politik haben kann und darf. So bleibt es zwar bei der kommissarischen Beaufsichtigung mitgliedstaatlicher Demokratien, die sich nun aber dadurch legitimieren soll, dass die Leitfigur der Aufsichtsbehörde demokratisch gewählt wird. Sollen wir wirklich glauben, dass die Bürger in den betroffenen Mitgliedstaaten, vor allem diejenigen, die von massiver Lohnsenkung und 
Schleifung sozialstaatlicher Sicherung existentiell betroffen sind - dies gehört bekanntlich zu den wesentlichen makroökonomischen Imperativen des „Regierens des Euro“ - davon wirklich zu beeindrucken sind? Wir befürchten demgegenüber, dass in dieser Konstellation ein Verweis auf eine neue „demokratische Legitimation“ des Kommissionpräsidenten von den betroffenen Bürgern, die sich in der heutigen Lage der Griechen, Portugiesen oder Spanier befinden, schlicht als Hohn empfunden würde.

Natürlich gibt es noch weitere Einwände gegen die (informelle) Direktwahl des Kommissionspräsidenten, die der Policy-Report nicht anspricht.

- Eine Wahl ohne Wahlrechtsgleichheit wird keine Akzeptanz finden, und zwar aus guten theoretischen Gründen, wenn denn der bislang gültige Grundsatz der rechtsstaatlichen Demokratietheorie, dass nämlich der Zwang des Rechts seine Legitimation aus der gleichberechtigen Teilhabe an der Genese des Rechts bezieht, nicht revidiert werden soll (oder wenn er denn revidiert werden soll, wie es allenthalben propagiert wird, wäre dies wenigstens einmal in theoria zu leisten).

- Die alteuropäische Demokratietradition sorgt sich vor allem um die rechtliche Bindung der Exekutive durch parlamentarisches Gesetz und steht darum der direkten Wahl exekutiver Spitzen generell skeptisch gegenüber. Die Bedenken gegenüber einer identitären Repräsentation durch eine Wahl der Spitze der Exekutive sind umso berechtigter, wenn die demokratischen Gegengewichte - Parlament, Parteien, Verbände, Öffentlichkeit - so schwach entwickelt sind wie in der Union.

- Unter den gegebenen Umständen würden zwar Konservative und Sozialdemokraten um das Amt des Kommissionpräsidenten konkurrieren. Aber die Wählerschaft würde sich nicht entlang der üblichen RechtsLinks-Achse organisieren, sondern entlang der Wohlstandsachse Nord-Süd. Schließlich ist - salopp gesprochen - nach Meinung auch vieler Sozialdemokraten Austeritätspolitik nicht schlecht, wenn sie die anderen trifft und die Haftungsrisiken des eigenen Mitgliedstaates zu senken verspricht. Statt demokratischer Legitimation würde die Direktwahl dann eine tiefe soziale Spaltung der Union ins Werk setzen, und der „demokratisch legitimierte“ Kommissionspräsident würde als nichts anderes denn als Repräsentant dieser Spaltung angesehen werden.

Wir sehen von einer Verlängerung dieser Reihe ab.

[1] Policy-Report "The Euro Crisis and the Democratic Governance of the Euro: Legal and Political Issues of a Fiscal Crisis”, vorgelegt für das High-Level Policy Seminar "The Democratic Governance of the Euro” am 10.5.2012 (alsbald verfügbar unter http://globalgovernanceprogramme.eui.eu/news-events/high-level-policy-seminars/thedemocratic-governance-of-the-euro/). Zu diesem Seminar wurden in einem Compiled Memorandum weitere Stellungnahmen veröffentlicht, darunter auch ein Beitrag von Ch. Joerges („The Economic Constitution in Crisis“).

Prof. Dr. Christian Joerges ist Direktor am Zentrum für Europäische Rechtspolitik und Professor für Deutsches und Europäisches Privat- und Wirtschaftsrecht an der Universität Bremen. Dr. Florian Rödl ist Forschungsgruppenleiter am Excellenzcluster "Die Herausbildung normativer Ordnungen" der Goethe-Universität Frankfurt/Main.

LICENSED UNDER CC BY NC ND SUGGESTED CITATION Joerges, Christian; Rödl, Florian: Direktwahl des EU-Kommissionspräsidenten: Mehr Schaden als Nutzen, VerfBlog, 2012/6/26, http://verfassungsblog.de/direktwahl-des-eukommissionsprsidentenmehr-schaden-als-nutzen/. 